\title{
Chemotherapy intensification for first-line treatment of poor- prognosis metastatic germ cell cancer is not yet ready for prime time
}

\author{
Di Maria Jiang, MD, MSC, FRCSC'; Robert J. Hamilton, MD, MPH, FRCSC2; \\ Aaron R. Hansen, BSc, MBBS (Hon), FRACP'
}

'Division of Medical Oncology and Haematology, Princess Margaret Cancer Centre, University of Toronto, Toronto, ON, Canada; ${ }^{2}$ Division of Urology, Department of Surgical Oncology, Princess Margaret Cancer Centre, University of Toronto, Toronto, ON, Canada

Cite as: Can Urol Assoc J 2020;14(2):48-9. hittp://dx.doi.org/10.5489/cuaj.6407

See related article on page 43

$\mathrm{T}$ he standard treatment for metastatic, poor-risk nonseminomatous germ cell tumor (NSGCT) based on International Germ Cell Cancer Collaborative Group (IGCCCG) risk stratification is four cycles of bleomycin, etoposide, cisplatin (BEP) or ifosfamide, etoposide, and cisplatin (VIP) chemotherapy in patients who are not suitable for bleomycin. Rate of tumor marker decline during initial chemotherapy is prognostic for survival and may help identify patients who may be resistant to standard chemotherapy. GETUG-13 was a phase 3, multicenter, randomized trial including patients with untreated, metastatic, poor-risk NSGCT. Following one cycle of BEP, marker decline kinetics, defined by a logarithmic formula, were calculated. Among 254 patients, 203 (80\%) with unfavorable decline (estimated time to normalization above pre-defined cutoffs or rising levels at cycle 2) were randomized to continue standard BEP for an additional three cycles, or switch to dosedense chemotherapy consisting of two cycles of paclitaxel, BEP, oxaliplatin (T-BEP-O) followed by two cycles of cisplatin, bleomycin, ifosfamide (PBI) with granulocyte colony stimulating factor support. The most recent data showed an improvement in five-year progression-free survival (PFS) rate (the primary endpoint) $-47 \%$ vs, $60 \%$ favoring dose-dense chemotherapy (hazard ratio [HR] $0.65 ; 95 \%$ confidence interval [CI] 0.43-0.97; $p=0.037) .{ }^{1}$ The five-year overall survival (OS) rate was improved, $61 \%$ vs. $70 \%$, but did not reach statistical significance (HR 0.69; 95\% Cl 0.43-1.11; $\mathrm{p}=0.12$ ). More severe adverse events, including neurotoxicity and nausea/vomiting, occurred in the dose-dense arm; however, renal and pulmonary toxicities, as well as rates of secondary malignancies were similar.

In this issue of CUAJ, Batra et al reported some important feasibility data with respect to implementing a chemotherapy intensification strategy analogous to the GETUG-13 protocol in the Canadian context. Among 10 patients with metastatic, poor-risk NSGCT, eight $(80 \%)$ had unfavorable tumor marker decline. The intensified chemotherapy used was T-BEP for three cycles followed by one cycle of paclitaxel, ifosfamide, cisplatin (TIP). In comparison with GETUG-13, major modifications included the omission of oxaliplatin from the two T-BEP-O cycles, and substitution of T-BEP for the first cycle of PBI and TIP for the second cycle of PBI. Of the six patients treated with intensified chemotherapy, two completed treatment as planned and four received cycle 4 cisplatin, etoposide, ifosfamide (PEI) instead of T-BEP due to decline in pulmonary function. At median follow up of 57.6 months, five patients were still alive (four had no evidence of disease, one had residual teratoma on surveillance), one died of synchronous metastatic adenocarcinoma ex teratoma, and none required salvage, highdose chemotherapy. These results seem aligned with results published from GETUG-13. Serious complications included febrile neutropenia $(n=3)$, grade 3 liver injury $(n=2)$, grade 3 anemia $(n=4)$, grade $3 / 4$ thrombocytopenia $(n=2)$, deep venous thrombosis requiring anticoagulation $(n=2)$, grade 3 neuropathy $(n=2)$, and grade 2 cognitive dysfunction $(n=1)$.

The total dose of bleomycin used by Batra et al in the intensified chemotherapy regimen was 270 units, much lower than the 520 units used in GETUG-13. Although dose reductions occurred in some patients in GETUG-13, the majority received all planned doses of bleomycin. While GETUG-13 specified a diffusing capacity corrected for alveolar volume (DLCO/VA) $<65 \%$ (or $\geq 10 \%$ over baseline) for holding bleomycin, Batra et al had a more conservative threshold of DLCO/VA $<75 \%$ (occurred in four patients), which further adds to under-dosing with bleomycin. This can potentially lead to suboptimal cure rates. The authors argue that the increased total dosages of etoposide $(1500 \mathrm{mg} /$ $\left.\mathrm{m}^{2}\right)$ and paclitaxel $\left(775 \mathrm{mg} / \mathrm{m}^{2}\right)$ compared to the GETUG-13 regimen $\left(1000 \mathrm{mg} / \mathrm{m}^{2}\right.$ and $350 \mathrm{mg} / \mathrm{m}^{2}$, respectively) should compensate for the under-dosage of bleomycin; however, robust supporting evidence for this strategy is lacking. Prior randomized trials evaluating high-dose chemotherapy 
(HDCT), including comparable doses of etoposide, did not demonstrate OS advantage over standard bleomycin-based regimens. ${ }^{2}$ Evidence supporting the use of paclitaxel $\geq 775$ $\mathrm{mg} / \mathrm{m}^{2}$ in poor-risk NSGCT is limited to single-arm studies, which showed severe prolonged myelosuppression ${ }^{3}$ and secondary malignancies. ${ }^{4}$

Overall, the strategy of chemotherapy intensification based on unfavorable marker decline has not been widely adopted worldwide. The lack of OS benefit is a significant limitation, and highlights the need to further improve patient selection and develop better treatment strategies in these patients. Other trials have also failed to show improved survival outcomes with upfront intensified chemotherapy in first-line treatment of metastatic, poor-risk NSGCT, with or without autologous stem cell support (ASCT). ${ }^{2,5}$ There are several potential reasons for the lack of OS benefit. Although tumor marker kinetics has been established as a prognostic factor, it is not predictive of treatment outcomes. In the GETUG-13 study, patients treated with standard BEP still reached five-year PFS and OS of $47 \%$ and $61 \%$, respectively, suggesting many patients can still be cured with upfront BEP despite unfavorable marker decline. Furthermore, while numerically more patients with unfavorable marker decline progressed on standard BEP, more patients underwent salvage HDCT plus ASCT in the BEP arm than in the dose-dense arm $(17 \%$ vs. $8 \%)$, some of which were likely cured with salvage therapy. This likely reduced the overall impact of dose-dense chemotherapy on OS. For patients who relapse, upfront dose-dense chemotherapy presumably decreases tolerability of salvage HDCT.

Potential over-treatment is a significant issue with chemotherapy intensification, since change in treatment was required in $80 \%$ of the patients. Select patients with very high tumor markers (e.g., hCG $500000 \mathrm{IU} / \mathrm{mL}$ ) may not ever achieve favorable marker decline according to timeto-normalization calculations. Unfortunately, there are no validated predictive biomarkers at this time, and the inability to predict treatment resistance is a major barrier of optimizing outcomes. However, genomic profiling holds promise for enabling precision treatment strategies. Presence of TP53 pathway alterations is strongly associated with cisplatin resistance and inferior outcomes. ${ }^{6}$ Other potential biomarkers of cisplatin resistance include high cytoplasmic p21 expression, ${ }^{7}$ increased DNA repair capacity, ${ }^{8}$ and presence of detectable microRNA in plasma. ${ }^{9}$ Even in patients with cisplatin-resistant disease, potentially actionable alterations were present in up to $55 \%$, including MDM2 amplification, RAS, KIT, FGFR3, AKT1, and PIK3CA alterations. ${ }^{6,10}$

Overall, Batra et al, in a retrospective, small, heterogeneous patient cohort, demonstrated feasibility of a modi- fied chemotherapy intensification approach for patients with metastatic, poor-risk NSGCT and unfavorable marker decline. The authors simplified the complicated GETUG13 regimen, which most likely contributed to the successful implementation of this complex approach. Novel biomarkers of cisplatin resistance should also be incorporated in future clinical trials evaluating chemotherapy intensification. Presumably, if such trials produce an improvement in OS, then chemotherapy intensification is more likely to be widely adopted as a standard of care.

Competing interests: Dr. Hansen has been a consultant and advisory board member for AstraZeneca Pharmaceuticals LP, Boehringer Ingelheim International GmbH, Boston Biomedical Inc., Bristol-Myers Squibb Company, GlaxoSmithKline Inc., Genetech Inc., Hoffmann La Roche Inc., Medlmmune LL, Merck Serono S.A., Novartis Pharmaceuticals Canada Inc., and Pfizer Inc.; and received a research grant from Karyopharm. The remaining authors reports no competing personal or financial interests related to this work.

\section{References}

1. Fizazi K, Flechon A, Le Teuff/ $G$, et al. Mature results of the GETUG 13 phase 3 trial in poor-prognosis germ-cell tumors (GCT). J Clin Oncol 2016;34:4504. https://doi.org/10.1200/JC0.2016.34.15_ suppl. 4504

2. Motzer RJ, Nichols CJ, Margolin KA, et al. Phase 3 randomized trial of conventional-dose chemotherapy with or without high-dose chemotherapy and autologous hematopoietic stem-cell rescue as first-line treatment for patients with poor-prognosis metastatic germ cell tumors. J Clin Oncol 2007;25:247-56. https://doi.org/10.1200/JC0.2005.05.4528

3. Hartmann JT, Gauler T, Metzner B, et al. Phase 1/2 study of sequential dose-intensified ifosfamide, cisplatin, and etoposide plus paclitaxel as induction chemotherapy for poor-prognosis germ cell tumors by the German Testicular Cancer Study Group. J Clin Med 2007;25:5742-7. https://doi.org/10.1200/ JC0.2007.11.9099

4. Feldman DR, Hu J, Dorff TB, et al. Paclitaxel, ifosfamide, and cisplatin efficacy for first-line treatment of patients with intermediate- or poor-risk germ cell tumors. J Clin Oncol 2016;34:2478-83. https://doi.org/10.1200/JC0.2016.66.7899

5. Daugaard G, Skoneczna I, Aass N, et al. A randomized, phase 3 study comparing standard-dose BEP with sequential high-dose cisplatin, etoposide, and ifosfamide (VIP) plus stem-cell support in males with poor-prognosis germ-cell cancer (EORTC 30974). Ann Oncol 2011122:1054-61. https://doi.org/10.1093/annonc/mdq575

6. Bagrodia A, Lee BH, Lee W, et al. Genetic determinants of cisplatin resistance in patients with advanced germ cell tumors. J Clin Oncol 2016;34:4000-7. https://doi.org/10.1200/JC0.2016.68.7798

7. Koster R, Gietema JA, Jong $S$ De, et al. Cytoplasmic $p 21$ expression levels determine cisplatin resistance in human testicular cancer. J Clin Invest 2010;120:3594-3605. htrps://doi.org/10.1172/JC141939

8. Köberle B, Masters JRW, Hartley JA, et al. Defective repair of cisplatin-induced DNA damage caused by reduced XPA protein in testicular germ cell tumors. Curr Biol 199;9:273-6. https://doi.org/10.1016/ S0960-9822(99)80118-3

9. Mego M, Agthoven T Van, Gronesova P, et al. Clinical utility of plasma miR-37 la-3p in germ cell tumors. $J$ Cell Mol Med 2019;23:1128-36. https://doi.org/10.1111/icmm.14013

10. Feldman DR, lyer $G$, Alstine $L$ Van, et al. Presence of somatic mutations within PIK3CA, AKT, RAS, and FGFR3 but not BRAF in cisplatin-resistant germ cell tumors. Clin Cancer Res 2014;14:3712-21. https://doi.org/10.1158/1078-0432.CCR-13-2868

Correspondence: Dr. Di Maria Jiang, Division of Medical Oncology and Haematology, Princess Margaret Cancer Centre, University of Toronto, Toronto, ON, Canada; Di.Jiang@uhn.ca 